\title{
Nonlinear dynamics and control of a recycle fixed bed reactor
}

Recke, Bodil; Jørgensen, Sten Bay

Published in:

Proceedings of the American Control Conference

Link to article, DOI:

10.1109/ACC.1997.608940

Publication date:

1997

Document Version

Publisher's PDF, also known as Version of record

Link back to DTU Orbit

Citation (APA):

Recke, B., \& Jørgensen, S. B. (1997). Nonlinear dynamics and control of a recycle fixed bed reactor. In Proceedings of the American Control Conference (Vol. 4, pp. 2218-2222). IEEE.

https://doi.org/10.1109/ACC.1997.608940

\section{General rights}

Copyright and moral rights for the publications made accessible in the public portal are retained by the authors and/or other copyright owners and it is a condition of accessing publications that users recognise and abide by the legal requirements associated with these rights.

- Users may download and print one copy of any publication from the public portal for the purpose of private study or research.

- You may not further distribute the material or use it for any profit-making activity or commercial gain

- You may freely distribute the URL identifying the publication in the public portal

If you believe that this document breaches copyright please contact us providing details, and we will remove access to the work immediately and investigate your claim 


\section{Nonlinear Dynamics and Control of a Recycle Fixed Bed Reactor}

\author{
Bodil O. Recke \\ Department of Chemical Engineering \\ Technical University of Denmark \\ DK2800 Lyngby, Denmark \\ br@kt.dtu.dk
}

\author{
Sten Bay Jørgensen \\ Department of Chemical Engineering \\ Technical University of Denmark \\ DK2800 Lyngby, Denmark \\ sbj@kt.dtu.dk
}

\begin{abstract}
The purpose of this paper is twofold. Primarily to describe the dynamic behaviour that can be observed in a fixed bed reactor with recycle of unconverted reactant. Secondly to describe the possibilities of model reduction in order to facilitate control design. Reactant recycle has been shown to introduce periodic solution to the fixed bed reactor, a phenomenon which is not seen for the system without the recycle, at least not within the Peclet number range investigated in the present work. The possibility of model reduction by the methods of modal decomposition, and by characteristics are investigated in the paper for the present case. Finally a criterion for actuator selection is formulated, and a simulated control example is given.
\end{abstract}

\section{Introduction}

The introduction of recycle on a process can improve the utilisation of raw materials and reduce energy consumption. Consequently there is an increased interest in operating processes in this favourable way. The literature on nonlinear dynamics of fixed bed reactors with recycle is rather limited. The type of recycle that is studied in this paper is mass recycle. This is accomplished by recycling unconverted reactant or a fraction of the outlet stream. Multiple periodic solutions have been reported in [3] and [10]. The periodic solutions are initiated by Hopf bifurcations, and it turns out that the periods in all cases are simple fractions of the thermal residence time. Investigation of dynamic behaviour in the case of energy recycle has been done in e.g. [6]. In this case the system can show periodic solutions as well as multiple steady states. Investigations of a combination of two recycle types are shown in e.g. [9] and [11]. Both report multiple steady states, however the two disagree on the existence of periodic solutions.

\section{Dynamic investigation}

In this context dynamic investigation is meant as an investigation of the type and number of solutions which can be expected for the system under investigation as some of the parameters of the system are changed. In short this is called a bifurcation analysis. In the present paper the bifurcation analysis is performed for the inlet conditions to the reactor $\left(x_{1, i n}, y_{i n}\right)$, since these are easily manipulated in subsequent experiments to verify the simulation results. The effect of including a dispersion term in the model, i.e. the effect of the Peclet numbers, is also investigated.

\section{1. $\quad$ Model}

The model of the fixed bed reactor with recycle used in the main part of this paper is given by the equations below. In the fixed bed reactor catalytic oxidation of hydrogen to form water is performed. Hydrogen is supplied to the reactor in wast excess, and consequently it is assumed that the reaction is a simple first order reaction with respect to the oxygen concentration and that the hydrogen concentration has no effect on the reaction rate. Arrhenius temperature dependence is applied. Furthermore all transport parameters are assumed constant, and radial effects are neglected. These assumption along with other minor ones give rise to the following pseudo homogeneous model, including a mass balance for oxygen, an energy balance, and finally a first order ordinary differential equation describing the recycle.

$$
\begin{aligned}
\varepsilon \frac{\partial x_{1}}{\partial t} & =-\kappa \frac{\partial x_{1}}{\partial z}+\frac{1}{\mathrm{Pe}_{m}} \frac{\partial^{2} x_{1}}{\partial z^{2}}-R \\
\frac{\partial y}{\partial t} & =-\kappa \frac{\partial y}{\partial z}+\frac{1}{\mathrm{Pe}_{h}} \frac{\partial^{2} y}{\partial z^{2}}+\operatorname{Bi}\left(y-y_{s}\right)+\beta R \\
r_{m} \frac{d x_{1, r e c}}{d t} & =x_{1, \text { out }}-x_{1, \text { rec }} \\
R & =\operatorname{Dm}_{1} e^{\gamma\left(1-\frac{1}{y}\right)}
\end{aligned}
$$

Boundary conditions :

$$
\begin{aligned}
\left.x\right|_{z=0} & =x_{1, \text { in }}+\alpha x_{1, \text { rec }}+\left.\frac{1}{\kappa \mathrm{Pe}_{m}} \frac{\partial x_{1}}{\partial z}\right|_{z \rightarrow 0^{+}} \\
\left.y\right|_{z=0} & =y_{i n}+\left.\frac{1}{\kappa \mathrm{Pe}_{h}} \frac{\partial y}{\partial z}\right|_{z \rightarrow 0^{+}} \\
\left.\frac{\partial x_{1}}{\partial z}\right|_{z=1} & =0 \\
\left.\frac{\partial y}{\partial z}\right|_{z=1} & =0
\end{aligned}
$$

For numerical values of the parameters see [10]. This model is then discretised using the method of orthogonal 


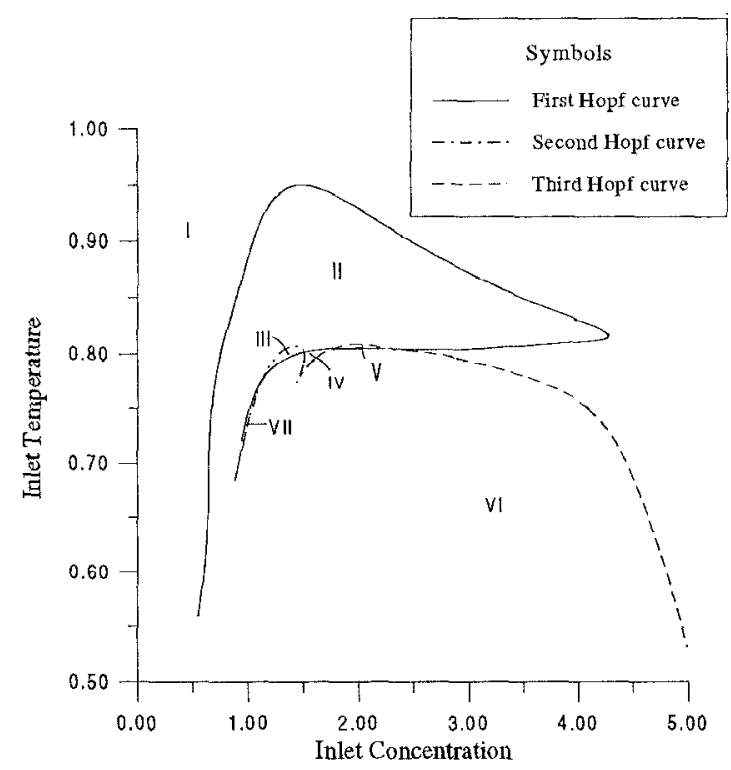

Figure 1: Bifurcation diagram for the fixed bed reactor with mass recycle (solution types see table 1)

collocation, as described in [14]. The resulting set of ordinary differential equations is then used in the bifurcation analysis. The analysis is performed using PATH [7].

\subsection{Bifurcation Diagram}

The types of bifurcations encountered in the present system are Hopf bifurcations and what will be defined as Hopf-like bifurcations. Hopf bifurcations are characterised by a migration of a pair of complex conjugate eigenvalues across the imaginary axis, as described in any textbook on nonlinear dynamics e.g. [5]. The main difference between Hopf and Hopf-like bifurcations is the dimension of the normal form necessary to determine the stability of the resulting periodic solution. In the case of an ordinary Hopf bifurcation a two dimensional normal form suffices. In contrast a Hopf-like bifurcation is characterised by having at least one eigenvalue with positive real part unaffected by the bifurcation, at least a three dimensional normal form is required. Furthermore the periodic solution arising from this type of bifurcation will always be unstable. A more in depth description of this type of bifurcation can be found in [10]. The bifurcation diagram shown in figure 1 is obtained when the inlet conditions are used as bifurcation parameters. The solution types in the various areas can be seen from table 1 .

The dynamic investigation shows that there is the possibility of three different periodic solutions, corresponding to migration into the right half complex plane of three different pairs of complex conjugate eigenvalues. On this basis the hypothesis is that the system can be described adequately by a low order model (ideally a sixth order
Table 1: Solution types in the areas numbered in figure 1

\begin{tabular}{|cccc|}
\hline area & stable ss & unstable ss & per. sol. \\
\hline I & 1 & - & - \\
II & - & 1 & 1 \\
III & - & 1 & 2 \\
IV & 1 & - & - \\
V & - & 1 & 2 \\
VI & - & 1 & 1 \\
VII & 1 & - & - \\
\hline
\end{tabular}

model). This hypothesis led to the investigation of the two different model reduction strategies described in the following section.

\section{Model reduction strategies}

In order to do realistic control design it is desirable to have a model of the process. Ideally this model should be as simple as possible, in order to make the design of the controller as easy as possible. When the process is described by partial differential cquations, the model is infinite dimensional, and this causes problems for the conventional control design strategies, since they are based on state space representations or at least a finite number of ordinary differential equations. However some strategies exist for making reductions in the case of partial differential equations. The two stratcgies that will be described in the following are the modal approximation and the method of characteristics.

\subsection{Modal approximation}

The first approach is a slightly modified version of the modal approximation described by e.g. Ray (1981)[8]. The modifications result partly from the fact that deviation variables are not used, and partly from the recycle equation. Because the modal approximation requires that the resulting system of coupled partial differential equation should be homogeneous in the boundary conditions. Thus the following approximations for the dimensionless oxygen concentration and temperature were applied.

$$
\begin{aligned}
x_{1}(z, t) & =\sum_{n=0}^{N} a_{1 n}(t) \phi_{n}(z)+x_{i n}+\alpha x_{1, r e c}(t) \\
y(z, t) & =\sum_{n=0}^{N} a_{2 n}(t) \psi_{n}(z)+y_{i n}
\end{aligned}
$$

These expansions, along with the assumption that the reaction term in the two partial equations can be written as an expansion in the eigenfunctions $\left(R=\sum_{n=0}^{N} f_{n} \phi_{n}\right)$, are introduced in the partial differential equations. This 
results in the following Sturm-Liouville problem.

$$
\begin{aligned}
-\kappa \phi_{n}^{\prime}+\frac{1}{\mathrm{Pe}_{m}} \phi_{n}^{\prime \prime} & =-\lambda_{1 n} \phi_{n} \\
-\kappa \psi_{n}^{\prime}+\frac{1}{\mathrm{Pe}_{h}} \psi_{n}^{\prime \prime} & =-\left(\lambda_{2 n}-\mathrm{Bi}\right) \psi_{n}
\end{aligned}
$$

Both equations have boundary conditions like

$$
\phi_{n}(0)-\frac{1}{\kappa \mathrm{Pe}_{m}} \phi_{n}^{\prime}(0)=0 \quad \phi_{n}^{\prime}(1)=0
$$

The coefficients $\lambda_{i n}$, can be determined from

$$
\lambda_{i n}=\frac{\alpha_{i n}^{2}}{\mathrm{Pe}_{m / h}}+\frac{\mathrm{Pe}_{m / h} \kappa^{2}}{4}
$$

where $\alpha_{i n}$ is the n'th eigenmode of the i'th equation, and can be calculated from

$$
\tan \left(\alpha_{i n}\right)=\frac{\alpha_{i n} \kappa \mathrm{Pe}_{m / h}}{\alpha_{i n}^{2}-\left(\frac{\kappa \mathrm{Pe}_{m / h}}{2}\right)^{2}}
$$

These equations can now be solved for the eigenfunctions $(\phi(z), \psi(z))$

$$
\begin{aligned}
& \phi_{n}(z)=e^{\frac{\kappa \mathrm{Pe}_{m} z}{2}} B_{1 n}\left(\frac{2 \alpha_{1 n}}{\kappa \mathrm{Pe}_{m}} \cos \left(\alpha_{1 n} z\right)+\sin \left(\alpha_{1 n} z\right)\right) \\
& \psi_{n}(z)=e^{\frac{\kappa \mathrm{Pe}_{h} z}{2}} B_{2 n}\left(\frac{2 \alpha_{2 n}}{\kappa \mathrm{Pe}_{h}} \cos \left(\alpha_{2 n} z\right)+\sin \left(\alpha_{2 n} z\right)\right)
\end{aligned}
$$

Where the coefficients $B_{i n}$ are determined in order to render the eigenfunctions orthogonal to the adjoint eigenfunctions (see e.g. [8] or [4]). With these results the original partial differential equations can now be reduced to a set of $2 N$ ordinary differential equations, where $N$ is the number of eigenmodes chosen to be included in the reduced model.

$$
\begin{aligned}
\varepsilon \frac{d a_{1 n}}{d t}+\lambda_{1 n} a_{1 n} & =-\operatorname{Dm} f_{1 n}-c_{n} \varepsilon \dot{x}_{1, r e c} \\
\frac{d a_{2 n}}{d t}+\left(\lambda_{1 n}-\mathrm{Bi}\right) a_{2 n} & =\beta \operatorname{Dm} f_{2 n}+\operatorname{Bid} d_{n}\left(y_{s}-y_{i} n\right)
\end{aligned}
$$

The coefficients are defined by

$$
\begin{aligned}
c_{n} & =\int_{0}^{1} \phi_{n}(z) d z \\
f_{1 n} & =\int_{0}^{1} x \exp \left(\gamma\left(1-\frac{1}{y}\right)\right) \phi_{n} d z
\end{aligned}
$$

and similar equations for $d_{n}$ and $f_{2 n}$ except that $\phi$ is exchanged by $\psi$. The equations (18)-(19) along with the original equation (3), can now be used to make a reduced order system, by only considering the dominant $N$ eigenmodes as significant. In principle this works very well, the only hiccup in the present case is in the numerical evaluation of the eigenfunctions. The problem is the term $e^{\frac{\kappa \mathrm{Pe}_{m} \underline{2}}{2}}$, since $\mathrm{Pe}_{m} \approx 270$ for this reactor model, there are great numerical difficulties in getting a reliable result in the present case. Consequently the method had to be abandoned. However it would work fine with lower Peclet numbers $(\mathrm{Pe} \lesssim 20$ ).

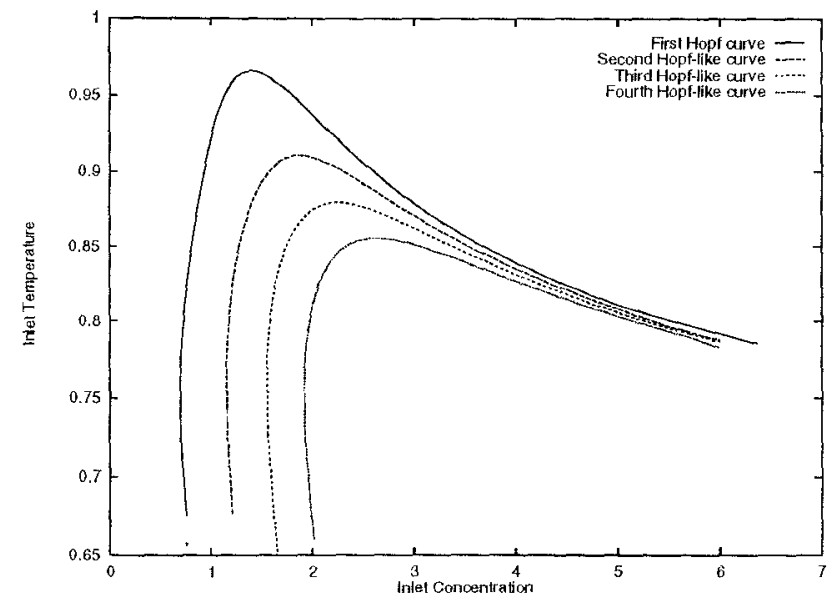

Figure 2: Bifurcation diagram for fixed bed reactor with mass recycle

\subsection{Method of characteristics}

In order to use the method of characteristics the partial differential equations have to be of first order in both space and time derivatives. This can be accomplished by neglecting the dispersion terms. Since the Peclet numbers are relatively large this approximation seems reasonable. However there are some fundamental differences in the system behaviour depending on whether dispersion is included or not. This can be seen when a bifurcation diagram like the one shown in figure 1 , is constructed. In the case of no dispersion the bifurcation diagram is shown in figure 2. A comparison of figures 1 and 2 clearly shows that there are differences in the dynamic behaviour depending on whether dispersion is included or not. In the case of no dispersion more unstable periodic solutions arise (there are even more Hopf-like bifurcation curves which are not shown in the figure). This means that the system most likely does not dampen out the high frequencies as when dispersion was included in the model. Consequently results obtained with a model without dispersion should always be evaluated critically to determine whether especially unstable periodic solutions are physically appearing in the system, or whether they are just a consequence of the modelling approximations. However in the present case when the model is to be used for control design, the approximation was not believed to be a serious problem. When the method of characteristics as described in e.g. [1], is applied to the present system, the following two characteristic directions result.

$$
\begin{aligned}
& \frac{d t}{d z}=\frac{1}{\kappa} \\
& \frac{d t}{d z}=\frac{\varepsilon}{\kappa}
\end{aligned}
$$


These directions are used for a change of variables defined by

$$
\begin{aligned}
& \frac{d t}{d \alpha}-\frac{1}{\kappa} \frac{d z}{d \alpha}=0 \\
& \frac{d t}{d \omega}-\frac{\varepsilon}{\kappa} \frac{d z}{d \omega}=0
\end{aligned}
$$

now the original equations can be rewritten as

$$
\begin{aligned}
& \frac{d x}{d \omega}=-\frac{x}{\varepsilon} \operatorname{Dm} \exp \left(\gamma\left(1-\frac{1}{y}\right)\right) \\
& \frac{d y}{d \alpha}=\beta x \operatorname{Dm} \exp \left(\gamma\left(1-\frac{1}{y}\right)\right)-\mathrm{Bi}\left(y-y_{s}\right)
\end{aligned}
$$

with boundary conditions

$$
\begin{aligned}
& \left.x\right|_{z=0}=x_{i n}+\left.\alpha x\right|_{z=1} \\
& \left.y\right|_{z=0}=y_{i n}
\end{aligned}
$$

As it can be seen the recycle is incorporated in the boundary conditions, along with the assumption that the recycle dynamics is pseudo stationary. The idea now is to make a mapping of e.g. output conditions. This corresponds to a discrete mapping, which maps one thermal residence time ahead. This means that the model is reduced to a set of two discrete equations. However there is a problem, since the recycle requires the knowledge of both inlet and outlet conditions in order to be solvable. If this point is ignored, the system is simply reduced to a normal fixed bed reactor. Another idea is to use two sets of discrete equations, one for the inlet and one for the outlet, in this way the recycle effect could be incorporated. But what happens at the outlet is of cause not independent of what happens at the inlet, and this coupling of the equations can not be taken into account with this approach. Consequently this method of model reduction was discarded as well.

\section{Control Design}

Before deciding on the particular control design and strategy to be applied in the specific case, it is important to be able to answer the question, what is the purpose of the control? Is it to extend the area in which the process can be operated with only one stable attractor (i.e. to avoid regions of periodic solutions), or perhaps to stabilise some of the open loop unstable periodic solutions or some third objective. An alternative approach could be to combine the two, i.e. to extend the area where a single stable steady state exists, and simultaneously ensure that the bifurcating periodic solution is stable. This approach is described in e.g. [15]. However this method applies nonlinear control technics and the calculations will be extremely time consuming if the model of the system is not of relatively low order and complexity.

The more simple approach taken in this paper, is to try to stabilise the unstable steady state in the areas where it is surrounded by either stable or unstable periodic solutions.
The controller applied is a simple PI-controller. The control parameters are designed with the aid of dynamic optimisation. The optimisations are performed using gOPT ([12], [13]) which is an optimisation algorithm integrated in the gPROMS system [2].

In designing controllers the first thing is to decide on actuators and measurements. The measurement chosen in the present case is the temperature at the outlet of the reactor (similar results will be obtained if concentration is used). The remaining problem is what actuator to choose? The idea is not to destroy the inherent feedback in the system, by breaking the recycle loop. Consequently candidate actuators are inlet temperature $\left(y_{i n}\right)$ and flow rate $(\kappa)$. However thorough investigation have shown that it seems impossible to stabilise the system at a steady state using any of these actuators, or indeed any other candidate actuators that will preserve the loop structure. This is probably caused by the fact that mass fluctuations travel much faster through the reactor than thermal ones does (confer $\varepsilon=1 / 600$ : ratio between mass and thermal residence time). Consequently the only possibility to obtain a fixed outlet temperature from the reactor is to break the recycle loop. i.e. to control the combined inlet concentration to the reactor $\left(x_{1, i n}+\alpha x_{1, r e c}\right)$. But it should be pointed out that from a dynamic point of view the system is no longer a recycle reactor, but behaves like an ordinary fixed bed reactor. An example of a closed loop simulation with a PI-controller designed by the objective function

$$
\min _{K_{p}} \int_{0}^{t_{f}}\left(y_{r e f}-y_{m}\right)^{4} d t
$$

is shown in figure 3 . The reason for the untraditional objective function, is to severely penalize oscillatory response. The latter is especially unattractive in the present case because of the inherent oscillatory nature of the open loop process. The change applied in the figure is a rather large set-point change. It can be seen from figure 3 that the controller is quite capable of handling even this large change, exactly as would be expected if the system had been a fixed bed reactor without recycle.

\section{Discussion and Conclusion}

It has been shown that multiple periodic solutions can arise in a fixed bed reactor with mass recycle. Furthermore a fundamental effect of dispersion in the model has been described. The main difference is that high frequency dynamics is damped in the case when dispersion is included and otherwise not. Not including dispersion will cause an increasing number of unstable periodic solutions to occur in the system, and since this is a known route to chaos, the system might behave chaotic for some parameter values.

Model reduction by either modal decomposition or the method of characteristics has been examined. But none of the methods gave results that were applicable in the 


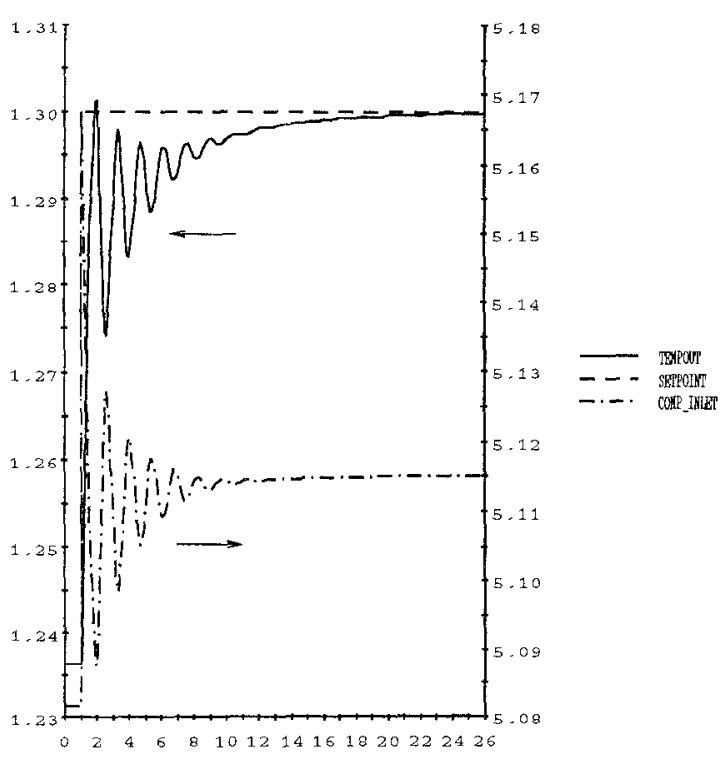

Figure 3: Stabilizing controller designed around $\left(x_{1, i n}, y_{i n}\right)=(1.0,0.8)$, control parameters $T_{i}=4.0$, $K_{p}=1.34726$

present case. The unsatisfactory result is caused by the high Peclet numbers, and the recycle respectively.

Finally it was shown that the only way to stabilise a fixed bed reactor with mass recycle at an open loop unstable steady state appears to be to break the natural feedback loop by controlling the combined inlet concentration. From a dynamic point of view, this results in a reduction of the system to an ordinary fixed bed reactor.

\section{References}

[1] R. Aris and N. R. Amundson. Mathematical Methods in Chemical Engineering, Volume 2 First-order partial differential equations with applications. PrenticeHall INC., Englewood Cliffs, New Jersey, 1973.

[2] P. I. Barton and C. C. Pantelides. The modelling of combined discrete/continuous processes. AIChE Journal, 40:966-979, 1994.

[3] H. Brabrand and S. B. Jørgensen. Dynamics of a packed bed reactor with reactant recycle. In $D Y$ CORD+'92. 3rd IFAC Symposium on Dynamics and Control of Chemical Reactors, Distillation Columns and Batch Processes, 1992.

[4] P. D. Christofides and P. Daoutidis. Nonlinear control of diffusion-convection-reaction processes. Computers chem. Engng., 20:Suppl.,S1071-S1076, 1996.

[5] J. Guckenheimer and P. Holmes. Nonlinear Oscillations, Dynamical Systems, and Bifurcations of Vector
Fields. Number 42 in Applied Mathematical Sciences. Springer-Verlag, New York, 1983.

[6] A. Kienle, G. Lauschke, V. Gehrke, and E.D. Gilles. On the dynamics of the circulation loop reactor- numerical methods and analysis. Chemical Engineering Science, 50:2361-2375, 1995.

[7] C. K. Petersen. PATH - user's guide. Department of Applied Mathematical Studies and Centre for Nonlinear Studies, University of Leeds, UK, 1989.

[8] W. H. Ray. Advanced process control. McGraw-Hill Book Company, 1981.

[9] B. Recke and S. B. Jørgensen. Physical implications of periodic solutions in a fixed bed reactor with recycle. In J. B. Rawlings, editor, DYCORD+'95. 4th IFAC Symposium on Dynamics and Control of Chemical Reactors, Distillation Columns and Batch Processes, 1995.

[10] B. O. Recke and S. B. Jørgensen. Nonlinear dynamics of a fixed bed reactor with recycle. In A.R. Giona G. Biardi, M. Giona, editor, Chaos and Fractals in Chemical Engineering. World Scientific Singapore, 1997.

[11] S. Subramanian and V. Balakotaiah. Classification of steady-state and dynamic behaviour of distributed reactor models. Chemical Engineering Science, 51:401$421,1996$.

[12] V. S. Vassiliadis, R. W. H. Sargent, and C. C. Pantelides. Solution of a class of multistage dynamic optimization problems. Part I - problems without path constraints. Ind. Eng. Chem. Res., 33:2111-2122, 1994.

[13] V. S. Vassiliadis, R. W. H. Sargent, and C. C. Pantelides. Solution of a class of multistage dynamic optimization problems. Part II - problems with path constraints. Ind. Eng. Chem. Res, 33:2123-2133, 1994.

[14] J. Villadsen and M. L. Michelsen. Solution of Differential Equation Models by Polynomial Approximation. Prentice Hall, 1978.

[15] H. O. Wang and E. H. Abed. Bifurcation control of a chaotic system. Automatica, 31:1213-1226, 1995. 